R. M. Huber

R. Fischer

\section{Endoluminale Brachytherapie mit Iridium 192 - eine zuwenig genutzte Therapieform für endobronchiale Tumoren}

\author{
Endoluminal Brachytherapy - an Underused Treatment Modality for \\ Endobronchial Tumours
}

Die Idee der Brachytherapie: Direkte Strahlenwirkung am Ort der Not unter Schonung des gesunden Gewebes. Im Vergleich zur externen, konventionellen Strahlentherapie bietet die endoluminale Brachytherapie durch den exponentiellen Dosisabfall den Vorteil, eine hohe Dosis nahe an das Tumorzielvolumen heranzubringen und dabei das umgebende Gewebe zu schonen.

Am stärksten verbreitet war und ist die Anwendung von Radium bei gynäkologischen Tumoren. Aber bereits zu Beginn des 20 . Jahrhunderts wurde in München die Therapie im Bronchialsystem mit Einlage radioaktiver Kugeln durchgeführt. In der Pneumologie fand dennoch die Brachytherapie anfangs keinen breiten Einsatz.

Dafür gab es zwei Gründe: Erstens waren aufgrund der niedrigen Aktivität der radioaktiven Quellen lange Anwendungszeiten für die Strahler notwendig, was für die Patienten unangenehm und durch die langen Liegezeiten auch gefährlich war. Zweitens bestand eine erhebliche radioaktive Belastung des Personals durch die radioaktiven Materialien.

Diese Probleme sind seit Mitte des 20. Jahrhunderts gelöst: Iridium 192 mit seiner hohen Dosisleistung ermöglicht die Applikation von hohen Strahlendosen in wenigen Minuten. Durch die Entwicklung geeigneter Applikatoren und der Nachladetechnik (Ausfahren des Strahlers aus einem Tresor nur während der unmittelbaren Therapiephase) ist optimaler Schutz für Patient und Anwender gewährleistet. Die Verwendung von computergestützten Simulationsprogrammen ermöglicht inzwischen auch die gleichzeitige Anwendung von mehreren Applikatoren zur räumlich geplanten Therapie. Damit ist eine gut geplante und hochwirksame Therapie mit zwei bis drei ambulanten Broncho- skopien möglich (Überblick in [1]). Dennoch wird die Brachytherapie in Deutschland im Vergleich zu anderen bronchologischen Therapieverfahren verhältnismäßig selten eingesetzt.

Diese Therapieoption kann aber bei allen symptomatischen Formen von endo- und peribronchialem Tumorwachstum in Betracht gezogen werden. In Bezug auf die langfristige Erhaltung der Atemwege und Verminderung der Symptome dürfte die endobronchiale Brachytherapie aufgrund ihres Wirkmechanismus im Vergleich mit anderen lokalen Verfahren am effektivsten sein. In einer kleinen randomisierten Studie verlängerte die endoluminale Kleinraumbestrahlung signifikant den Effekt der NdYAG-Lasertherapie mit Verringerung von zusätzlichen bronchoskopischen Eingriffen [2]. In den meisten publizierten Studien zur palliativen Brachytherapie wurde eine Verbesserung der Symptome bei 65 - $95 \%$ aller Patienten gezeigt.

Macha u. Mitarb. [3] beschreiben in der vorliegenden Ausgabe der Pneumologie im Rezidiv nach externer Bestrahlung erneut die Effektivität dieser Behandlungsmethode. In der Literatur kontrovers diskutiert wird die Problematik der massiven, tödlichen Blutungen. Hier konnten die Autoren zeigen, dass die an Hämoptoe verstorbenen Brachytherapiepatienten im Median 10,2 Monate länger leben als die gematchten Kontrollen. In einer holländischen Analyse von Blutungen zeigte sich bei alleiniger Strahlentherapie eine der Brachytherapie vergleichbare Blutungsrate. Erst ab einer Dosis pro Sitzung von $15 \mathrm{~Gy}$ auf $10 \mathrm{~mm}$ Abstand von der Strahlenquelle erhöhte sich das Blutungsrisiko durch die Brachytherapie [4]. In einer randomisierten Studie konnte umgekehrt gezeigt werden, dass die Reduktion der Dosis pro Sitzung von 7,5 Gy auf 4 Gy auf $10 \mathrm{~mm}$ keine weitere Reduktion der Blutungsraten bewirkte [5]. Man darf aus den bisher 
publizierten Daten wohl schlussfolgern, dass die endoluminale Brachytherapie bei einer Einzeldosis unter $15 \mathrm{~Gy}$ (auf $10 \mathrm{~mm}$ ) keine erhöhte Rate an akuten tödlichen Blutungen zur Folge hat und dass die späteren Blutungen als Folge des längeren Überlebens und des Fortschreitens des Tumorleidens anzusehen sind.

Die endoluminale Hochdosis-Brachytherapie mit Iridium 192 hat einen tumorspezifischen und lang anhaltenden Effekt. Kleine Tumoren können damit sogar geheilt werden. Darüber hinaus ist eine Kombination mit allen anderen lokalen und systemischen Tumortherapien möglich. Sie stellt zwar eine permanente interdisziplinäre Herausforderung dar mit engem Kontakt zwischen Radioonkologen und Pneumologen, verdient aber einen breiteren Einsatz in der pneumologischen Onkologie.

\section{Literatur}

${ }^{1}$ Fischer R, Pollinger B, Huber RM. Endobronchiale Brachytherapie. Dtsch Med Wochenschr 2000; 125: 1539-1541

${ }^{2}$ Chella A, Ambrogi MC, Ribechini A et al. Combined Nd-YAG-laser/HDR brachytherapy versus Nd-YAG-laser only in malignant central airway involvement: a prospective randomized study. Lung Cancer 2000; 27: $169-175$

${ }^{3}$ Macha HN, Bach P, Wahlers B et al. Überleben und Todesursachen nach palliativer endobronchialer Brachytherapie mit Iridium 192-High dose bei rezidivierendem Bronchialkarzinom. Pneumologie 2005; 59: $12-17$

${ }^{4}$ Langendijk JA, Tjwa MK, de Jong JM et al. Massive haemoptysis after radiotherapy in inoperable non-small cell lung carcinoma: is endobronchial brachytherapy really a risk factor? Radiother Oncol 1998; 49: $175-183$

${ }^{5}$ Huber RM, Fischer R, Hautmann $\mathrm{H}$ et al. Palliative endobronchial brachytherapy for central lung tumors. A prospective, randomized comparison of two fractionation schedules. Chest 1995; 107: 463-470 